\title{
PATHOPHYSIOLOGY OF FETAL HYDROCEPHALUS IN A DEVELOPMENTAL RAT MODEL OF SPINA BIFIDA
}

\author{
L. Beni-Adani \\ Pediatric Neurosurgery, Dana Children's Hospital, Tel-Aviv, Israel
}

Objectives: The two hit hypothesis claims that on top of the first hit of non-closure of the neural tube, secondary damage occurs during the rest of gestation leading to additional significant morbidity in fetuses with Meningomyelocele (MMC). The objective of the study was to understand the pathophysiology of MMC-associated Hydrocephalus (HCP) in a fetal developmental model of MMC in rats.

Materials and methods: This study was conducted as part of a research project focused on the secondary neuronal damage occurring during gestation in fetuses with MMC. The study was performed in collaboration with the Fetal treatment center at UCSF. MMC was created by administration of Retinoic-Acid (RA) to ratmothers during neuro-embryogenesis. At embryonal days 15-19 fetuses were removed and CSF pathways were studied in vivo to evaluate ventricular dilatation and pattern of major CSF pathway obstruction.

Results: of 16 fetuses with MMC only 2 had significant ventriculomegaly with blockage at foramen magnum. Other MMC fetuses and all control fetuses demonstrated passage of dye from the injected lateral ventricle to the contralateral, third, fourth ventricles and down through the central canal. 14/16 MMC fetuses showed extravasation of the dye around the interrupted arachnoid at MMC site with no obstruction of major CSF pathways.

Conclusions: Obstructive HCP is rare in fetuses with MMC. Abnormal absorption (communicating HCP) may be the major reason for persistent HCP even in the absence or reversal of chiari 2 . This is the first report of in-vivo documentation of obstructive HCP in fetuses with MMC. 